\title{
Solvatochromic Effects on the Absorption Spectrum of 2-Thiocytosine
}

\author{
Sebastian Mai, ${ }^{\dagger}$ Brennan Ashwood, ${ }^{\ddagger}$ Philipp Marquetand, ${ }^{\dagger}$ Carlos E. Crespo-Hernández,,$* \ddagger \odot$ \\ and Leticia González ${ }^{*} \dagger \oplus$
}

\begin{abstract}
${ }^{\dagger}$ Institute of Theoretical Chemistry, Faculty of Chemistry, University of Vienna, Währinger Strasse 17, 1090 Vienna, Austria
${ }^{\ddagger}$ Center for Chemical Dynamics and Department of Chemistry, Case Western Reserve University, 10900 Euclid Avenue, Cleveland, Ohio 44106, United States
\end{abstract}

\section{Supporting Information}

ABSTRACT: The solvatochromic effects of six different solvents on the UV absorption spectrum of 2-thiocytosine have been studied by a combination of experimental and theoretical techniques. The steady-state absorption spectra show significant shifts of the absorption bands, where in more polar solvents the first absorption maximum shifts to higher transition energies and the second maximum to lower energies. The observed solvatochromic shifts have been rationalized using three popular solvatochromic scales and with high-level multireference quantum chemistry calculations including implicit and explicit solvent effects. It has been found that the dipole moments of the excited states account for some general shifts in the excitation energies,

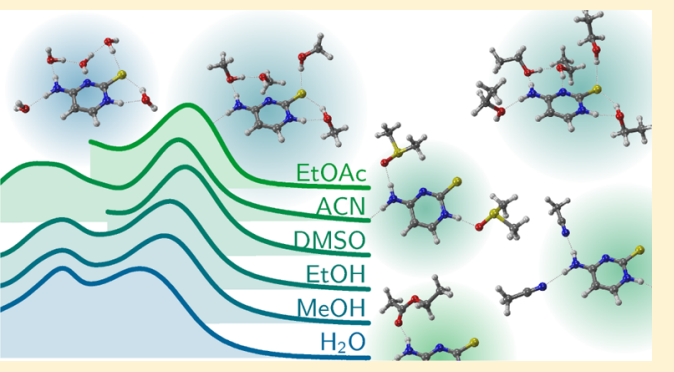
whereas the explicit solvent interactions explain the differences in the spectra recorded in the different solvents.

\section{INTRODUCTION}

Thiobases are a class of nucleobase analogues, molecules that are structurally similar to the canonical nucleobases found in DNA. They differ from the canonical nucleobases only by the substitution of carbonyl oxygen atoms by sulfur atoms, and this chemical similarity makes the thiobases important nucleobase analogues. For instance, thiobases are widely used in biological and medical research to investigate the genetic code ${ }^{1}$ and in chemo- $^{2-4}$ and antiviral therapies. ${ }^{4,5} 2$-Thiocytosine (2tCyt), as well as other thiobases, is naturally found in bacterial $t$-RNA. ${ }^{6-8}$ In addition, the photochemical properties of thiobases are exploited for applications such as photolabeling, ${ }^{9,10}$ photoaffinity probing of nucleic acid structures and DNA/RNAprotein interactions, ${ }^{10,11}$ or photochemotherapeutic applications. $^{12-15}$

Two major characteristics explain the photochemical behavior of thiobases. ${ }^{16,17}$ First, the absorption spectra of the thiobases are significantly red-shifted compared to the ones of the canonical nucleobases. Second, UVA excitation of each thiobase leads to near unity population of long-lived triplet states through ultrafast intersystem crossing, ${ }^{16}$ contrasting the efficient relaxation mechanisms exhibited by the canonical nucleobases. ${ }^{18,19}$ The remarkably different photochemical properties of the thiobases compared to the nucleobases have recently been rationalized as due to the differential stabilization of key regions of the excited-state potential energy surfaces upon sulfur substitution. ${ }^{17}$

The particular microscopic environment that the thiobases encounter in solution, or when incorporated into DNA or RNA, is expected to significantly affect the energies of the electronic states and their absorption properties. This sensitivity of the electronic states on the surroundings makes thiobases interesting candidates for photolabeling agents. In this regard, it is desirable to obtain a detailed understanding of the solvatochromic properties of thiobases, i.e., how the solvent hydrogen bonding, polarity, or polarizability actually contribute to the shifts of the main absorption bands. This knowledge is also important for interpreting the absorption shifts in a biological environment, which is less polar than many solvents, ${ }^{20}$ but where hydrogen bonds often play a crucial role.

Of particular relevance to this work, the absorption spectrum of 2 tCyt is only known in aqueous solution,,$^{16,17,21-24}$ and only recently its lowest-energy absorption band was assigned to particular electronic states. ${ }^{17,24}$ On the contrary, a notable number of publications report ab initio calculations on the tautomeric ratio of $2 \mathrm{tCyt}$ in gas phase, solid phase, and aqueous solution. $^{25-32}$

In this contribution, we specifically investigate the effect of polar aprotic and protic solvents on the absorption spectrum of 2 tCyt. We present steady-state absorption spectra in six different solvents: ethyl acetate (EtOAc), acetonitrile (ACN), dimethyl sulfoxide (DMSO), ethanol (EtOH), methanol $(\mathrm{MeOH})$, and aqueous phosphate-buffered saline solution $\left(\mathrm{H}_{2} \mathrm{O}\right)$. This set of solvents excludes low-polarity solvents, due to the low solubility of $2 \mathrm{tCyt}$ in such solvents. Moreover, other solvents in which $2 \mathrm{tCyt}$ is soluble absorb in the same spectral range as $2 \mathrm{tCyt}$, and therefore were also excluded from the study. The energetic positions of the absorption maxima are

Received: March 22, 2017

Revised: April 27, 2017

Published: April 28, 2017 
subjected to solvatochromic analyses ${ }^{33-35}$ to decompose the solvent shifts into contributions from different solvent effects. The experimental results are complemented with high-level multistate complete active space second order perturbation theory (MS-CASPT2) calculations, which provide a rigorous assignment of the absorption bands and rationalize the influence of the aprotic and protic solvents on the vertical electronic transitions. The knowledge gained is fundamental to understand the excited-state dynamics and photochemistry of 2 tCyt when incorporated into DNA or RNA, and to evaluate its prospective use as a photolabeling agent.

\section{METHODS}

Experimental Methods. 2-Thiocytosine (Sigma-Aldrich, 97\%), sodium dihydrogen phosphate (Sigma-Aldrich, 99.0\%), and sodium hydrogen phosphate (Sigma-Aldrich, 99.0\%) were used as received. Phosphate buffered saline solution was prepared using $0.15 \mathrm{~g}$ of sodium dihydrogen phosphate and $0.27 \mathrm{~g}$ of sodium hydrogen phosphate dissolved in $200 \mathrm{~mL}$ of ultrapure water to give $\mathrm{pH} 7.4$ aqueous phosphate buffer with a total phosphate concentration of $16 \mathrm{mM}$. All other solvents were used as received: acetonitrile (Fisher Scientific, 99.9\%), methanol (Fisher Scientific, 99.9\%), ethanol (Fisher Scientific, 99.8\%), dimethyl sulfoxide (Sigma-Aldrich, 99.9\%), and ethyl acetate (Fisher Scientific, 99.9\%).

The steady-state absorption spectra were recorded using a Cary 100 Bio UV-vis Spectrophotometer (Varian) in $1 \mathrm{~cm}$ optical path quartz cells (Starna Cells, Inc.) and were corrected for the background signal of the solvent.

Computational Methods. The ab initio calculations performed correspond to three main tasks: (i) the evaluation of the most stable tautomer, (ii) high-level reference computations of the vertical excitation energies in vacuum, and (iii) vertical excitation calculations considering implicit and explicit solvent effects of the six solvents used in the experiments.

For the first task, namely the evaluation of the most stable tautomer of $2 \mathrm{tCyt}$ in vacuum and in solution, we performed RI$\mathrm{MP2} /$ cc-pVTZ $^{36-38}$ optimizations/frequency calculations and $\mathrm{RI}-\mathrm{CCSD}(\mathrm{T}) / \mathrm{cc}-\mathrm{pVTZ}$ single point calculations. At this stage, we considered only implicit solvent effects, by means of the COSMO (conductor-like screening model) ${ }^{39}$ solvation model, as implemented in Orca 3.0. ${ }^{40}$

For the second task, i.e., the high-level reference calculation in vacuum, we performed MS-CASPT2 $(14,10) /$ ANO-RCCVQZP computations including 9 electronic states, ${ }^{41-43}$ based on state-averaged (SA9) complete active space self-consistent field (SA-CASSCF) wave functions. The active space, shown in Figure 1 , contains the full $\pi$ system ( 8 orbitals) plus the lone pairs of sulfur and the aromatic nitrogen atom $\left(n_{\mathrm{S}}\right.$ and $n_{\mathrm{N}}$ in Figure 1). The Douglas-Kroll-Hess (DKH) Hamiltonian, ${ }^{44}$ Cholesky decomposition, ${ }^{45}$ and the default IPEA shift ${ }^{46,47}$ of 0.25 a.u. were employed, but no level shift was applied. These calculations were performed with MOLCAS 8.0. ${ }^{48}$ The geometry for this reference gas-phase calculation was calculated with RI-MP2/cc-pVQZ using Orca 3.0. ${ }^{36-38}$

For the third task, explicit and implicit solvent effects were included in the vertical excitation computations. This was accomplished by placing three to five solvent molecules close to 2tCyt (microsolvation) in positions that facilitate hydrogen bonding, mimicking the hydrogen bond network reported by Podolyan et al. ${ }^{31}$ For EtOAc, ACN, and DMSO, solvent placement considered only the possible acceptor-donor

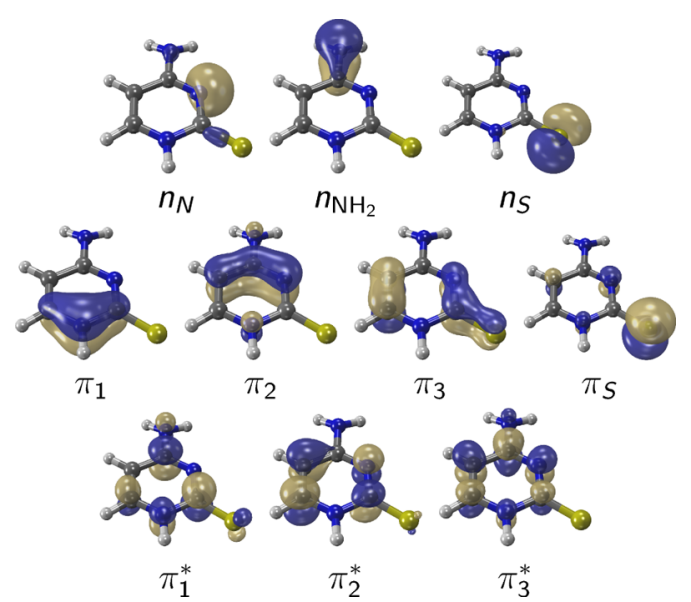

Figure 1. Active space orbitals of $2 \mathrm{tCyt}$ with naming convention.

hydrogen bonds, leading to the inclusion of three solvent molecules close to the $\mathrm{N}_{1}-\mathrm{H}$ and $\mathrm{NH}_{2}$ groups. For EtOH, $\mathrm{MeOH}$, and $\mathrm{H}_{2} \mathrm{O}$, the positions of the solvent molecules also considered donor-acceptor hydrogen bonding, leading to two more solvent molecules near the $\mathrm{N}_{3}$ and $\mathrm{S}$ atoms. These structures (i.e., 2tCyt plus solvent molecules) were then optimized at the BP86/aug-cc-pVDZ ${ }^{37,38,49}$ level of theory, using COSMO to account for the effect of the bulk solvent. These optimizations were performed with Orca 3.0, ${ }^{40}$ except for the structure microsolvated with $\mathrm{ACN}$, which was optimized with Turbomole $7.0{ }^{50}$ whose optimizer better handled the linear ACN molecules. Due to the choice of the positions of the solvent molecules, these optimized geometries favor hydrogen bonding over dipolar or dispersion interactions, which is justified in view that $2 \mathrm{tCyt}$ is readily soluble in protic solvents.

Using the optimized, microsolvated geometries, we performed SA9-CASSCF $(14,10) /$ cc-pVDZ $^{37,38}$ calculations followed by MS-CASPT2 $(14,10)^{41}$ calculations for the vertical excitation energies. The effect of the bulk solvent was added by means of PCM (polarizable continuum model) ${ }^{51-53}$ in the dielectric version implemented in MOLCAS 8.0 (see Table S1 in the Supporting Information (SI) for the employed solvent parameters). The PCM charges were first equilibrated with the ground state density in a single-state CASSCF computation, whereas in the SA calculations only the dynamical part of the reaction field was adapted to the electron density (nonequilibrium calculation). Instead of employing the SA density, ${ }^{52}$ we performed 9 independent SA-CASSCF + MS-CASPT2 calculations $(i=1, \ldots, 9)$, such that the density of state $i$ was used in calculation $i$ (RASSI state overlaps were used to check that no root flipping occurred). Finally, only the energies and oscillator strengths (from the perturbatively modified CASCI wave functions ${ }^{41}$ ) of state $i$ were taken from calculation $i$, while the other states were neglected. In this way, each state's density is in equilibrium with the dynamical part of the reaction field, and it is not necessary to apply a state-specific correction as, e.g., in ref 52 .

Since the microsolvated structures involve relatively many atoms and PCM-MS-CASPT2 is quite expensive, it was necessary to decrease the size of the basis set used in these calculations to double- $\zeta$. As discussed in the SI (Figure S1), the smaller basis set leads to a decrease of all excitation energies by about $0.17 \mathrm{eV}$, but otherwise produces energies in close agreement with the quadruple- $\zeta$ basis set, with a standard deviation of only $0.08 \mathrm{eV}$. Although this standard deviation is 
larger than some shifts between similar solvents (e.g., EtOH and $\mathrm{MeOH}$, see below), it can be assumed that the shifts due to the basis set are similar in all solvents, leading to error cancellation in the solvent effects. For these reasons, we are confident that the reduced basis set size does not affect the conclusions from our solvation computations. In the calculations, the DKH Hamiltonian, Cholesky decomposition, and the active space shown in Figure 1 were employed. The IPEA shift was set to zero, as this is expected to provide better results in combination with a double- $\zeta$ basis set. ${ }^{47}$ To avoid intruder states, an imaginary level shift ${ }^{54}$ of 0.3 a.u. was needed. MOLCAS $8.0^{48}$ was employed for the MS-CASPT2 calculations.

The so-obtained excitation energies are here directly compared to the experimental absorption maxima, or the energetic positions of fitted Gaussians (see below). This is a simplification, because the vibrational broadening can induce systematic shifts of the absorption maximum relative to the vertical excitation energy, which were reported to be on the order of $0.1-0.3 \mathrm{eV}^{55}$ However, under the assumption that in the different solvents these systematic shifts are similar, our main conclusions concerning the solvatochromic effects should still hold.

\section{EXPERIMENTAL RESULTS}

Stationary Absorption Spectrum. Figure 2 shows the absorptivity spectra of $2 \mathrm{tCyt}$ in the six solvents considered, with

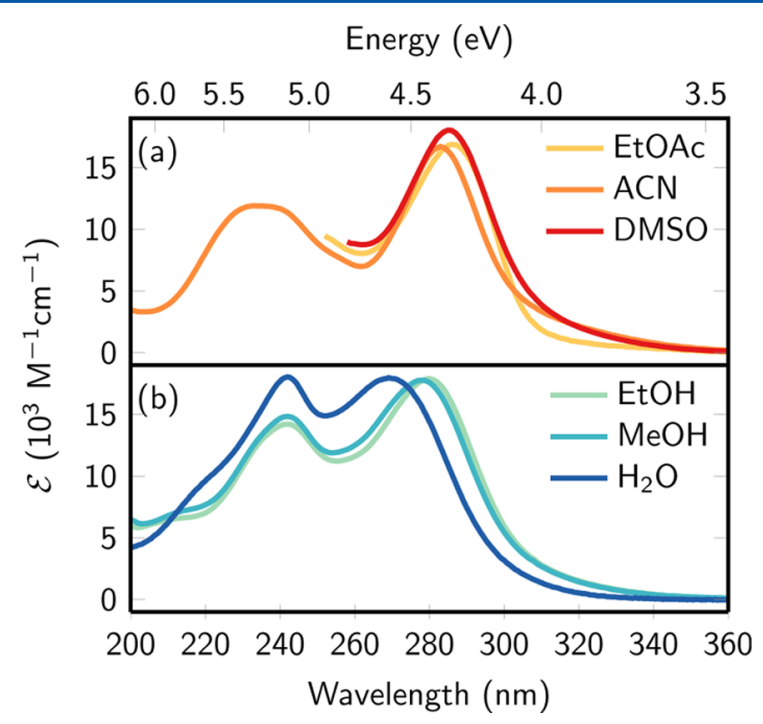

Figure 2. Absorptivity spectra of the lowest-energy absorption bands of $2 \mathrm{tCyt}$ in the considered solvents, with aprotic solvents in (a) and protic solvents in (b). The spectra of EtOAc and DMSO was not recorded at wavelengths shorter than ca. $250 \mathrm{~nm}$ due to strong solvent absorption.

the wavelengths and absorptivities given in Table 1 . The spectra show an intense absorption maximum at $269-286 \mathrm{~nm}$ (4.3-4.6 $\mathrm{eV}$ ), with a residual absorption tail extending to approximately 330-350 nm (3.7-3.9 eV), depending on the solvent used. A second absorption band with a maximum at 233-242 nm $(5.1-5.3 \mathrm{eV})$ can be observed in $\mathrm{ACN}, \mathrm{EtOH}, \mathrm{MeOH}$, and $\mathrm{H}_{2} \mathrm{O}$. In addition, the protic solvents (EtOH, $\mathrm{MeOH}, \mathrm{H}_{2} \mathrm{O}$ ) give rise to a shoulder in the $210-218 \mathrm{~nm}(5.6-5.9 \mathrm{eV})$ range. These observations generally agree with the absorption spectra previously reported in $\mathrm{H}_{2} \mathrm{O}$ and $\mathrm{ACN}^{21,23,24}$
A number of solvatochromic trends can be observed in the spectra, especially when going from polar aprotic solvents to polar protic solvents. On the one hand, the lowest-energy maximum is blue-shifted by $17 \mathrm{~nm}$ in going from EtOAc (286 $\mathrm{nm})$ to $\mathrm{H}_{2} \mathrm{O}(269 \mathrm{~nm})$, while the molar extinction coefficient remains approximately constant. On the other hand, the highenergy maximum is red-shifted by $9 \mathrm{~nm}$, from $233 \mathrm{~nm}$ in ACN to $242 \mathrm{~nm}$ in $\mathrm{H}_{2} \mathrm{O}$, with significant narrowing and an increase in the molar extinction coefficient. In addition, a shoulder develops in the protic solvents, located at approximately 210 $\mathrm{nm}$ in the alcohols and at $218 \mathrm{~nm}$ in $\mathrm{H}_{2} \mathrm{O}$. Finally, the absorption tail at low energies blue-shifts in protic solvents, especially in $\mathrm{H}_{2} \mathrm{O}$, as has been observed in other thiobases. ${ }^{16}$

Figure 3 presents least-squares fits of a sum of five Gaussians to the experimental absorption spectra. This number of Gaussians was found to satisfactorily describe the four observed spectral features (the absorption tail, two maxima, one shoulder) and additionally the absorption at around $200 \mathrm{~nm}$, which is the edge of the recorded spectral range. For EtOAc and DMSO, only three Gaussians were necessary, because the spectral features below $250 \mathrm{~nm}$ are concealed by the absorption of these solvents. The relevant equation, the fit parameters obtained (intensities, central energies, full width at halfmaximum, and corresponding fitting errors of the five Gaussians, Table S2), and the residuals from the fits (Figure S2) are given in the SI.

As shown in Figure 3, in all solvents the absorption spectrum of $2 \mathrm{tCyt}$ is dominated by the lowest-energy absorption band, which is well described by a single, intense Gaussian $\left(g_{2}\right)$. The energetic position of this Gaussian notably blue-shifts when going from the least polar solvent, EtOAc, to the most polar solvent, $\mathrm{H}_{2} \mathrm{O}$. In general, it appears that the absorption tail (described by $g_{1}$ ) follows the shifts of the lowest-energy absorption maximum, i.e., the tail blue-shifts when going from the less polar solvents to $\mathrm{H}_{2} \mathrm{O}$; however, the behavior of $g_{1}$ is slightly more complex than the one of $g_{2}$. In particular, the spectrum in ACN shows the most pronounced absorption tail, extending to $360 \mathrm{~nm}$ (chosen cutoff wavelength where the absorptivity is still $200 \mathrm{M}^{-1} \mathrm{~cm}^{-1}$ ), whereas the tails in EtOAc, DMSO, $\mathrm{MeOH}$, and $\mathrm{EtOH}$ extend to $350 \mathrm{~nm}$ and that in $\mathrm{H}_{2} \mathrm{O}$ to only $330 \mathrm{~nm}$. According to the Gaussian fits, the absorption tails in ACN and DMSO show the strongest absorption (largest intensities for $g_{1}$ ), whereas the tail is weaker in the other four solvents. We note here that this complex behavior might be partially because it is difficult to unambiguously fit $g_{1}$-in some cases, an almost equally good fit could be obtained by simultaneously blue-shifting, increase of width, and increase of intensity, so that the shape of the absorption tail is approximately conserved.

The decomposition of the high-energy absorption band in Figure 3 for $\mathrm{ACN}, \mathrm{EtOH}, \mathrm{MeOH}$, and $\mathrm{H}_{2} \mathrm{O}$ shows that the high-energy absorption band and its shoulder can be described well with two Gaussians ( $g_{3}$ and $\left.g_{4}\right)$, although the fitting uncertainties were slightly larger than for $g_{1}$ and $g_{2}$. The position and intensity ratio of $g_{3}$ and $g_{4}$ depends on the solvent, especially in $\mathrm{EtOH}$ and $\mathrm{MeOH}$, where the $g_{4}$ component becomes very small.

Solvatochromic Analyses. We performed solvatochromic analyses using three widely employed linear solvent effect scales, proposed by Catalán, ${ }^{33}$ Kamlet and $\mathrm{Taft}^{34}$ and Reichardt, ${ }^{35}$ respectively. Because the Catalán method is the most recent one and-through its four parameters-allows decomposing the solvent shifts into different contributions, we 
Table 1. Experimental Wavelengths $\lambda(\mathrm{nm})$, Energies $E(\mathrm{eV})$, and Absorptivities $\mathcal{E}\left(\mathrm{M}^{-1} \mathrm{~cm}^{-1}\right)$ of Absorption Maxima and Shoulders in Figure 2

\begin{tabular}{|c|c|c|c|c|c|c|c|c|c|}
\hline \multirow[b]{2}{*}{ solvent } & \multicolumn{3}{|c|}{ first maximum } & \multicolumn{3}{|c|}{ second maximum } & \multicolumn{3}{|c|}{ shoulder } \\
\hline & $\lambda$ & $E$ & $\mathcal{E}$ & $\lambda$ & $E$ & $\mathcal{E}$ & $\lambda$ & $E$ & $\mathcal{E}$ \\
\hline EtOAc & 286 & 4.33 & 16870 & & & & & & \\
\hline $\mathrm{ACN}$ & 283 & 4.38 & 16700 & 233 & 5.32 & 11910 & & & \\
\hline DMSO & 285 & 4.35 & 18030 & & & & & & \\
\hline $\mathrm{EtOH}$ & 280 & 4.43 & 17900 & 242 & 5.12 & 14220 & $\sim 210$ & 5.90 & 6500 \\
\hline $\mathrm{MeOH}$ & 278 & 4.46 & 17770 & 242 & 5.12 & 14840 & $\sim 212$ & 5.84 & 7050 \\
\hline $\mathrm{H}_{2} \mathrm{O}$ & 269 & 4.61 & 17950 & 242 & 5.12 & 18030 & $\sim 218$ & 5.68 & 9000 \\
\hline
\end{tabular}

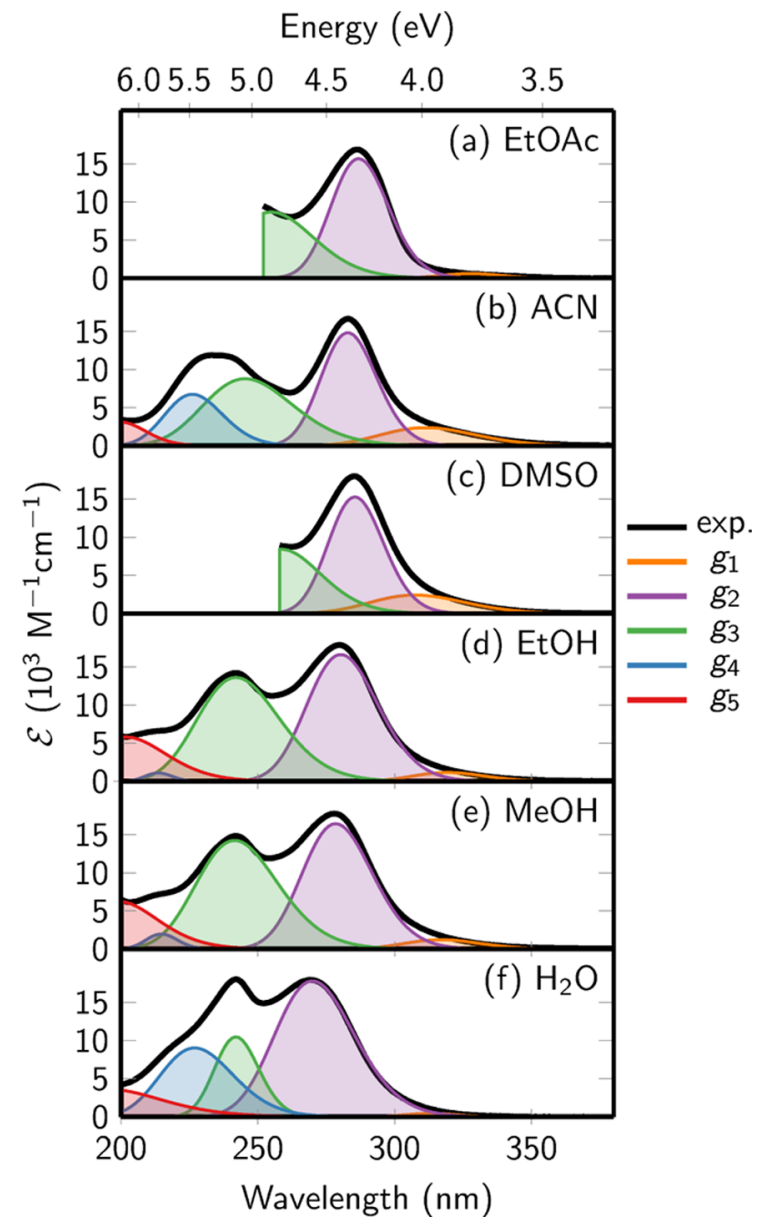

Figure 3. Absorptivity spectra of 2tCyt in various solvents (black lines) and decompositions of the spectra into a sum of Gaussians $g_{1}$ to $g_{5}$.

focus our discussion mostly on this solvatochromic method. The employed numerical data (absorption energies, solvent parameters, see Tables S3-S6) and the results of the three analyses are given in the SI.

The solvatochromic analyses were carried out for the energies of the first absorption maximum and absorption tail in the different solvents. We focus on these two spectral features because the underlying transitions play key roles in the photophysics of 2tCyt, but also because in DMSO and EtOAc the high-energy band is overshadowed by the strong absorption of the solvents below $250 \mathrm{~nm}$ and thus we cannot determine the energetic position of this band.

In the Catalán analysis, the solvent-dependent energy shifts of the absorption bands are linearly related to four solvatochromic parameters, which Catalan calls the solvent acidity SA, solvent basicity SB, solvent dipolarity SdP, and solvent polarizability SP. ${ }^{33}$ Out of the 15 linear regression models possible with four parameters, the statistically most significant $(p<0.001)$ models for the absorption maximum energies are "only SA": $E_{\max }=(4.34 \pm 0.01)+(0.24 \pm 0.02)$ $S A$ with $R^{2}=0.96$, and "SA+SB": $E_{\max }=(4.39 \pm 0.02)+(0.21$ $\pm 0.02) \mathrm{SA}+(-0.09 \pm 0.03) \mathrm{SB}$ with $R^{2}=0.99$. Including the SdP and SP parameters in the models did not lead to statistically significant improvements of the fits. These results show that the solvent acidity and basicity (SA and SB) alone can successfully describe the solvatochromic shifts of the lowest-energy absorption maximum observed in Figure 2, whereas solvent dipolarity and polarizability do not have a notable effect. However, it should be remarked that the maximum position could also be explained solely with the solvent acidity.

For the red absorption tail, the linear regression models based on the Catalán parameters do not lead to conclusive results. The best-performing model, "only SA", gives $R^{2}=0.81$. Models with more parameters were not found to be statistically significant. As the magnitudes of the absorption coefficients for the red tail are small and also the solvent data set is limited, we conclude that it is unwarranted to perform further solvatochromic analyses for the red absorption tail.

The Kamlet-Taft analysis (see SI) showed that the most relevant solvent parameter for the absorption maximum is the hydrogen bond donor acidity $\alpha,{ }^{56}$ in agreement with the Catalán analysis. The two other Kamlet-Taft parameters, solvent polarizability and hydrogen bond acceptor basicity, seem to only play a minor role for the solvent shifts of the absorption maximum. The Reichardt $E_{\mathrm{T}}^{\mathrm{N}}$ parameter (see SI), which is mostly related to solvent polarity and acidity, ${ }^{57}$ was found to correlate well with the absorption maximum energies, however, being a single-parameter model it does not allow identifying the most influential solvent property.

\section{COMPUTATIONAL RESULTS}

Next, we present computational results to identify the lowestenergy tautomer in each of the different solvents, to assign the deconvoluted absorption spectra in Figure 3, and to rationalize the observed solvatochromic behavior.

Tautomer Ratios. Table 2 shows the relative energies of different tautomers (displayed in Figure 4) of 2tCyt in vacuum and in the six solvents used in this work. Interestingly, the amino-thiol form with its two rotamers (B and C) is the most stable tautomer in the gas phase, where other tautomers are at least $5 \mathrm{kcal} / \mathrm{mol}$ higher in energy. Conversely, the $1 \mathrm{H}$-aminothion tautomer is the most stable form in all the solvents considered here and is predicted to be the only tautomer in $\mathrm{ACN}, \mathrm{DMSO}, \mathrm{EtOH}, \mathrm{MeOH}$, and water. However, it is possible that the amino-thiol form may be present in a few percent when using EtOAc as solvent. The vast abundance of 
Table 2. Relative Energies ( $\mathrm{kcal} / \mathrm{mol}$ ) and Permanent Dipole Moments (Debye) of 2tCyt Tautomers (Shown in Figure 4) in Different Solvents ${ }^{a}$

\begin{tabular}{lccccc} 
& $\mathrm{A}$ & $\mathrm{B}$ & $\mathrm{C}$ & $\mathrm{D}$ & $\mathrm{E}$ \\
\hline vacuum & 5.0 & $\mathbf{0 . 0}$ & $\mathbf{0 . 2}$ & 5.6 & 12.1 \\
EtOAc & $\mathbf{0 . 0}$ & 2.1 & 2.1 & 5.1 & 6.2 \\
$\mathrm{ACN}$ & $\mathbf{0 . 0}$ & 4.6 & 4.6 & 6.8 & 4.9 \\
$\mathrm{DMSO}$ & $\mathbf{0 . 0}$ & 4.8 & 4.7 & 6.9 & 4.9 \\
$\mathrm{EtOH}$ & $\mathbf{0 . 0}$ & 4.3 & 4.3 & 6.6 & 4.9 \\
$\mathrm{MeOH}$ & $\mathbf{0 . 0}$ & 4.5 & 4.5 & 6.8 & 5.0 \\
$\mathrm{H}_{2} \mathrm{O}$ & $\mathbf{0 . 0}$ & 5.0 & 4.9 & 7.1 & 4.8 \\
$\mu_{\text {vac }}$ & 7.3 & 3.6 & 4.3 & 5.0 & 8.3 \\
${ }^{a}$ Level of theory: & $\mathrm{CCSD}(\mathrm{T}) / \mathrm{cc}-\mathrm{pVTZ} / / \mathrm{MP} 2 / \mathrm{cc}-\mathrm{pVTZ}+\mathrm{COSMO}$.
\end{tabular}

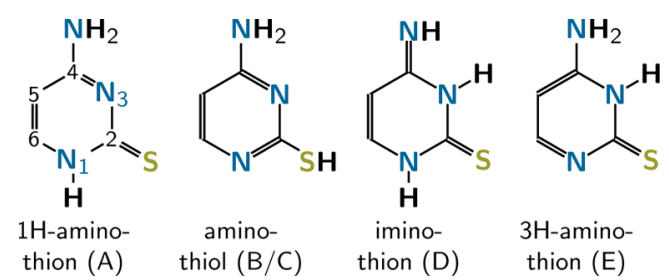

Figure 4. Structures of the investigated tautomers of $2 \mathrm{tCyt}$, with ring atom numbering.

the $1 \mathrm{H}$-amino-thion form is due to its large permanent dipole moment (7.3 D in vacuum, according to $\operatorname{CCSD}(\mathrm{T}) / \mathrm{cc}-\mathrm{pVTZ}$ ), which leads to a large stabilization in both the polar protic and polar aprotic solvents studied. These results agree satisfactorily with the findings reported in the literature, $25,26,29,31$ which consistently predict the $1 \mathrm{H}$-amino-thion tautomer to be the most stable one in water. Based on our findings, we focus our attention on how the vertical excitation energies for the $1 \mathrm{H}$ amino-thion tautomer (simply referred to as 2tCyt hereafter) are affected by these solvents.

Vertical Excitation Energies in Vacuum. Table 3 compiles the gas-phase vertical excitation energies, oscillator

Table 3. Vertical Excitation Energies, Oscillator Strengths, and Wave Function Characters of the Lowest Eight Singlet States of $2 \mathrm{tCyt}^{a}$

$\begin{array}{ccclc}\text { state } & E(\mathrm{eV}) & f_{\text {osc }} & \text { char. } & \mu(\mathrm{D})^{b} \\ S_{0} & 0.00 & & \mathrm{GS} & 7.5 \\ S_{1} & 3.65 & 0.001 & n_{\mathrm{S}} \pi^{*} & 2.9 \\ S_{2} & 3.74 & 0.055 & \pi_{\mathrm{S}} \pi^{*} & 3.2 \\ S_{3} & 3.98 & 0.000 & n_{\mathrm{S}} \pi^{*} & 1.7 \\ S_{4} & 4.44 & 0.657 & \pi_{\mathrm{S}} \pi^{*} & 6.3 \\ S_{5} & 5.24 & 0.003 & n_{\mathrm{N}} \pi^{*} & 4.8 \\ S_{6} & 5.60 & 0.292 & \pi \pi^{*} & 7.7 \\ S_{7} & 5.78 & 0.005 & n_{\mathrm{N}} \pi^{*} & 9.1 \\ S_{8} & 6.10 & 0.139 & \pi \pi^{*} & 9.7\end{array}$

${ }^{a} \mathrm{MS}$ (9)-CASPT2 (14,10)/ANO-RCC-VQZP//RI-MP2/cc-pVQZ（in vacuum). ${ }^{b}$ Dipole moments from SS-CASPT2.

strengths, and dipole moments for the $1 \mathrm{H}$-amino-thion tautomer of $2 \mathrm{tCyt}$ obtained at the MS-CASPT2/quadruple- $\zeta$ level of theory. The four lowest excited singlet states are excitations from the thiocarbonyl group - two states with $n_{\mathrm{S}} \pi^{*}$ character $\left(S_{1}\right.$ and $\left.S_{3}\right)$ and two states with $\pi_{\mathrm{S}} \pi^{*}$ character $\left(S_{2}\right.$ and $\left.S_{4}\right)$; refer to Figure 1 for the involved orbitals. Higher-lying states originate from excitation from the nitrogen lone pair $\left(n_{\mathrm{N}} \pi^{*}\right)$ and from excitation within the pyrimidine $\pi$ system $\left(\pi \pi^{*}\right)$ according to the MS-CASPT2 calculations. Based on the calculated oscillator strengths, the most intense transitions are the $\pi_{\mathrm{S}} \pi^{*}\left(S_{4}\right)$ and $\pi \pi^{*}\left(S_{6}, S_{8}\right)$ states, with energies of $4.44 \mathrm{eV}$ $(279 \mathrm{~nm}), 5.60 \mathrm{eV}(221 \mathrm{~nm})$, and $6.10 \mathrm{eV}(203 \mathrm{~nm})$, respectively. The lowest $\pi_{\mathrm{S}} \pi^{*}$ state $\left(S_{2}\right)$, at $3.74 \mathrm{eV}(331 \mathrm{~nm})$, shows weaker absorption than the other $\pi \pi^{*}$ states, with an oscillator strength that is an order of magnitude smaller than that of the $S_{4}$ state. The $n_{\mathrm{S}} \pi^{*}$ and $n_{\mathrm{N}} \pi^{*}$ states are dark (i.e., oscillator strength smaller than 0.01 ) according to the calculations.

Table 3 also reports the norms of the permanent dipole moment vectors of the excited states, which are expected to influence the energy shifts in solution. Based on these data, the states $S_{1}$ to $S_{5}$ are all less polar than the ground state of $2 \mathrm{tCyt}$. On the contrary, the higher state $S_{6}$ has approximately the same dipole moment as the ground state, and the $S_{7}$ and $S_{8}$ states are more polar than the ground state.

Solvation Effects on the Computed Vertical Excitations. We now present the results of the MS-CASPT2 calculations incorporating solvent effects. The optimized microsolvated geometries are shown in Figure 5, giving the positions of the explicit solvent molecules. Based on the optimized structures, a significant solvent effect on the groundstate equilibrium geometry of $2 \mathrm{tCyt}$ was observed. Most importantly, the $\mathrm{S}=\mathrm{C}_{2}-\mathrm{N}_{3}=\mathrm{C}_{4}-\mathrm{N}$ moiety undergoes bond length alteration in polar solvents: the $\mathrm{C}_{2}=S(+0.05 \AA)$ and $\mathrm{N}_{3}=\mathrm{C}_{4}(+0.03 \AA)$ bonds become longer, whereas the $\mathrm{N}_{1}-\mathrm{C}_{2}$ and $\mathrm{C}_{4}-\mathrm{N}_{\text {amino }}$ bonds become shorter $(-0.03 \AA)$; see Table S7 in the SI for more information. The effects of these geometric changes on the excitation energies will be discussed below.

Table 4 presents the results of the computed vertical excitations incorporating both explicit and implicit solvent effects. The table also includes (in column "vac") calculations in vacuum with otherwise identical settings for comparison (note that these values are thus different from those in Table 3; see Figure S3 for a comparison of the two vacuum computations). A number of trends can be observed in these results. The three lowest excited states $S_{1}\left(n_{\mathrm{S}} \pi^{*}\right), S_{2}\left(\pi_{\mathrm{S}} \pi^{*}\right)$, and $S_{3}\left(n_{\mathrm{S}} \pi^{*}\right)$ are significantly blue-shifted in solution, but their oscillator strengths remain small and are weakly affected by solvation. The oscillator strength of the $S_{2}\left(\pi_{\mathrm{S}} \pi^{*}\right)$ state is modulated between 0.01 and 0.03 , but without any particular trend. These modulations are due to slightly different mixing of the $S_{2}$ with the very bright $S_{4}$, which leads to different extent of intensity borrowing. The bright $S_{4}\left(\pi_{\mathrm{S}} \pi^{*}\right)$ state is blue-shifted from 4.3 $\mathrm{eV}(288 \mathrm{~nm})$ in vacuum to $4.6 \mathrm{eV}(269 \mathrm{~nm})$ in water and its oscillator strength is reduced from 0.7 to 0.4 . The $n_{\mathrm{N}} \pi^{*}$ states $\left(S_{5}\right.$ and $\left.S_{7}\right)$ are also slightly blue-shifted but remain dark in all solvents. Finally, the bright $\pi \pi^{*}$ states $\left(S_{6}\right.$ and $\left.S_{8}\right)$ red-shift and become brighter when going from polar aprotic to polar protic solvents.

\section{DISCUSSION}

In this section, we discuss the solvatochromic effects on the lowest-energy absorption maximum and the assignment of the experimental transitions in the absorption spectra of $2 \mathrm{tCyt}$ based on the results obtained from the vertical excitation energies calculated at the MS-CASPT2 level of theory.

Experimental Absorption Spectra. The results of the three solvatochromic analyses, using the Catalán, ${ }^{33}$ KamletTaft, $^{34}$ and Reichardt ${ }^{35}$ solvent scales, indicate that mainly the solvent acidity determines the energetic position of the first absorption maximum. This finding explains the large differences 

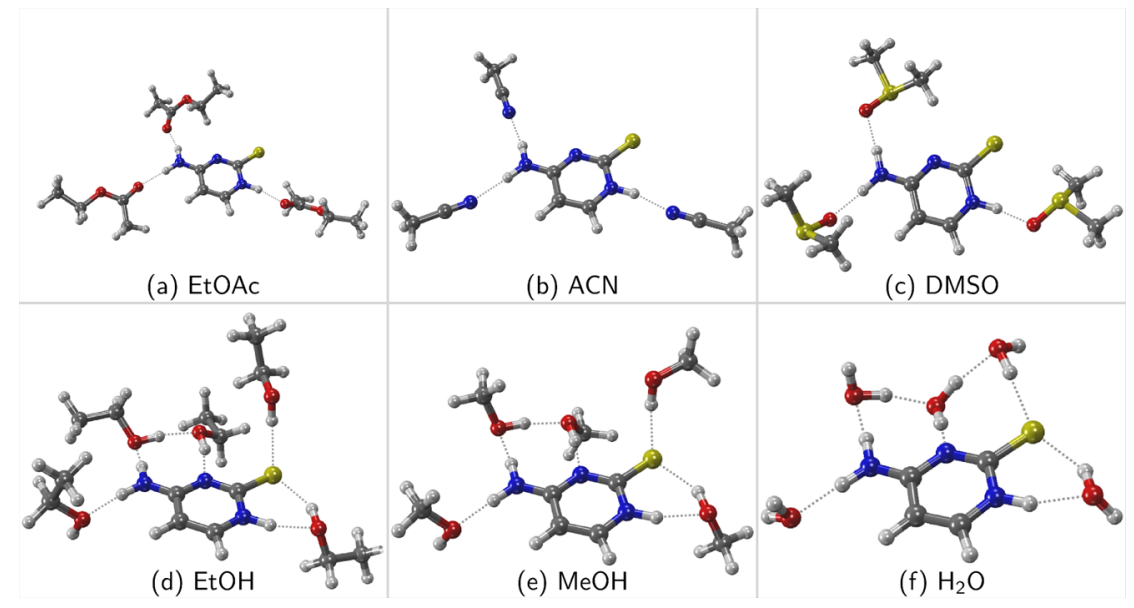

Figure 5. Optimized geometries of $2 \mathrm{tCyt}$ microsolvated with different number of solvent molecules, as depicted. Optimized with BP86/aug-ccpVDZ+COSMO. For coordinates, see the SI.

Table 4. Vertical Excitation Energies and Oscillator Strengths of 2tCyt in Various Solvents at the MS-CASPT2 Level of Theory ${ }^{a}$

\begin{tabular}{|c|c|c|c|c|c|c|c|c|c|}
\hline state & char. & $\mathrm{vac}$ & EtOAc & $\mathrm{ACN}$ & DMSO & $\mathrm{EtOH}$ & $\mathrm{MeOH}$ & $\mathrm{H}_{2} \mathrm{O}$ & \\
\hline \multicolumn{10}{|c|}{ energies $(\mathrm{eV})$} \\
\hline$S_{1}$ & $n_{\mathrm{S}} \pi^{*}$ & 3.46 & 3.93 & 4.01 & 4.01 & 4.33 & 4.36 & 4.50 & strong up \\
\hline$S_{2}$ & $\pi_{\mathrm{S}} \pi^{*}$ & 3.47 & 3.93 & 3.99 & 4.04 & 4.21 & 4.24 & 4.23 & strong up \\
\hline$S_{3}$ & $n_{\mathrm{S}} \pi^{*}$ & 3.77 & 4.32 & 4.42 & 4.51 & 4.94 & 4.97 & 5.12 & strong up \\
\hline$S_{4}$ & $\pi_{\mathrm{S}} \pi^{*}$ & 4.31 & 4.42 & 4.49 & 4.46 & 4.58 & 4.59 & 4.60 & up \\
\hline$S_{5}$ & $n_{\mathrm{N}} \pi^{*}$ & 5.07 & 5.29 & 5.37 & 5.42 & 5.76 & 5.78 & 5.72 & up \\
\hline$S_{6}$ & $\pi \pi^{*}$ & 5.17 & 4.84 & 4.88 & 4.72 & 4.81 & 4.82 & 4.81 & down \\
\hline$S_{7}$ & $n_{\mathrm{N}} \pi^{*}$ & 5.66 & 5.51 & 5.59 & 5.52 & 5.80 & 5.81 & 5.79 & up \\
\hline$S_{8}$ & $\pi \pi^{*}$ & 5.70 & 5.25 & 5.30 & 5.15 & 5.07 & 5.08 & 5.14 & down \\
\hline \multicolumn{10}{|c|}{ oscillator strengths } \\
\hline$S_{1}$ & $n_{\mathrm{S}} \pi^{*}$ & 0.00 & 0.00 & 0.00 & 0.00 & 0.00 & 0.00 & 0.02 & \\
\hline$S_{2}$ & $\pi_{\mathrm{S}} \pi^{*}$ & 0.02 & 0.01 & 0.02 & 0.02 & 0.03 & 0.03 & 0.01 & \\
\hline$S_{3}$ & $n_{\mathrm{S}} \pi^{*}$ & 0.00 & 0.00 & 0.00 & 0.01 & 0.02 & 0.01 & 0.02 & \\
\hline$S_{4}$ & $\pi_{\mathrm{S}} \pi^{*}$ & 0.69 & 0.59 & 0.58 & 0.56 & 0.48 & 0.47 & 0.40 & darker \\
\hline$S_{5}$ & $n_{\mathrm{N}} \pi^{*}$ & 0.00 & 0.00 & 0.00 & 0.00 & 0.00 & 0.00 & 0.02 & \\
\hline$S_{6}$ & $\pi \pi^{*}$ & 0.24 & 0.27 & 0.31 & 0.23 & 0.24 & 0.23 & 0.31 & brighter \\
\hline$S_{7}$ & $n_{\mathrm{N}} \pi^{*}$ & 0.00 & 0.01 & 0.01 & 0.01 & 0.01 & 0.01 & 0.01 & \\
\hline$S_{8}$ & $\pi \pi^{*}$ & 0.16 & 0.33 & 0.28 & 0.39 & 0.46 & 0.50 & 0.45 & brighter \\
\hline
\end{tabular}

${ }^{a}$ Note that for all columns the same MS-CASPT2 settings (MS-CASPT2(14,10)/cc-pVDZ+PCM // BP86/aug-cc-pvdz+COSMO) were used and therefore the values given here for "vac" differ from the ones in Table 3.

between aprotic and protic solvents displayed in Figure 2. On the contrary, the solvent polarizability or hydrogen bond acceptor strength do not seem to affect the absorption energy significantly.

Theoretical Absorption Spectra in Vacuum. We now discuss the high-level reference excited-state calculations of 2 tCyt in vacuum and compare the results to the experimental absorption spectra. From the onset, the calculated tautomer energies in Table 2 clearly show that the $1 \mathrm{H}$-amino-thion form should be the most abundant tautomer present in all the solvents investigated. This finding considerably simplifies the assignment of the absorption bands in the spectrum of $2 \mathrm{tCyt}$. In vacuum, the tautomeric equilibrium shifts to the amino-thiol form, but since we did not record an absorption spectrum in the gas phase, this tautomer is not relevant to our analysis. Still, the vacuum calculations for the $1 \mathrm{H}$-amino-thion tautomer alone should provide a general representation of the set of relevant excited states and their properties, while simultaneously helping to scrutinize the accuracy of the calculations that take into consideration explicit and implicit solvent effects.
The vacuum MS-CASPT2 calculations (Table 3) show that only some particular states have significant oscillator strengths and contribute to the lowest-energy absorption bands in Figure 2. These states either show dominant involvement of the $\pi_{S}$ orbital on the sulfur atom $\left(S_{2}, S_{4}\right)$ or of the $\pi$ orbitals localized on the pyrimidine ring $\left(S_{6}, S_{8}\right)$. Together, these states should give rise to two absorption maxima, one near $4.4 \mathrm{eV}\left(S_{4}\right)$ with a tail $\left(S_{2}\right)$, and another near 5.6-6.1 eV $\left(S_{6}, S_{8}\right)$ in the gas phase.

The calculations also yielded the norms of the permanent dipole moment vectors of the excited states (see Table 3), which are expected to influence the energy shifts when going from vacuum to solution. In particular, states with a dipole moment larger than the one of the ground state (about 7.5 D) should red-shift in polar solvents, whereas states with smaller dipole moments will blue-shift. For $2 \mathrm{tCyt}$, the states $S_{1}$ to $S_{5}$ are all less polar than the ground state, implying that these states should be shifted to higher energies in polar solvents. Interestingly, this also includes the bright $\pi_{S} \pi^{*}\left(S_{4}\right)$ state, even though states of $\pi \pi^{*}$ character are usually red-shifted in polar solvents. On the contrary, the higher states $S_{6}$ to $S_{8}$ are 
more polar than the ground state and can be expected to redshift upon solvation.

Despite the fact that the reference calculation does not include solvent effects, the transition energies $(4.4 \mathrm{eV}, 5.6 \mathrm{eV}$, $6.1 \mathrm{eV})$ compare-perhaps coincidentally-well with the absorption spectra recorded in EtOAc, ACN, or DMSO, which present a band centered at $4.3-4.4 \mathrm{eV}$ with a tail and a broad band at 5.1-5.4 eV. The protic solvents perturb the electronic transitions to a larger extent than $\mathrm{ACN}$, and hence the calculations performed in vacuum do not compare well with the experimental absorption spectra in these solvents. This observation is consistent with the solvatochromic analyses, which showed that solvent acidity plays a large role in the solvent shifts.

Theoretical Absorption Spectra in Solution. In order to better understand the influence of solvation on the absorption spectra, here we disentangle the different effects of the solvent on the vertical excitation energies of $2 \mathrm{tCyt}$ presented in Table 4. We consider the following effects: (i) change of molecular geometry, (ii) change of dielectric constant $\epsilon$, (iii) change of index of refraction $n$ (which is related to the dielectric constant at high frequencies $\epsilon_{\infty}=n^{2}$ ), and (iv) explicit solute-solvent interactions through hydrogen bonds. Figure 6 shows the results of the decomposition analysis. The four panels of the figure are organized such that the four effects are switched on one by one, in the above order, to yield the full solvation calculations in the last panel.

In Figure 6a, we investigate the effect of the structural changes from the equilibrium geometry in vacuum to the equilibrium geometry in aqueous solution (the optimized geometry from Figure 5f). The geometry in water was employed because the optimized geometries in the other solvents are located somewhere between the vacuum geometry and the water one. The changes observed are mainly related to bond length alteration of the $\mathrm{S}=\mathrm{C}_{2}-\mathrm{N}_{3}=\mathrm{C}_{4}-\mathrm{N}$ moiety (see Figure 4 for atom numbering). The panel shows that the geometric change of $2 \mathrm{tCyt}$ alone leads to a strong red-shift of the $S_{1}, S_{2}$, and $S_{3}$ states, a weak red-shift of the $S_{4}$ and $S_{5}$ states, virtually no shift of the $S_{6}$ and $S_{7}$ states, and a blue-shift of the $S_{8}$ state. These shifts are fully opposite to what is observed in the experiment and in the full solvation calculations (as shown in Table 4). Thus, the geometric relaxation of $2 \mathrm{tCyt}$ due to solvation alone cannot explain the trends in the experimental spectra. Instead, it is counteracted by the interactions of the excited-state dipole moments with the solvent, as well as by specific solute-solvent interactions.

In Figure 6b, we modify the dielectric constant $\epsilon$ used in the PCM model from 1 (vacuum) to 80 (water). Because for this panel we use the geometry optimized in water, the leftmost points of panel (b) are identical to the rightmost points of panel (a), with both panels forming a continuous scan. The increase of $\epsilon$ leads to the expected result that less polar states (especially $S_{1}, S_{2}$, and $S_{3}$ ) are shifted to higher energies, whereas the polar ${ }^{1} \pi \pi^{*}$ states are shifted to lower energies. This shows that part of the solvatochromic shifts observed experimentally originate from the interaction of the solute dipole moment with the solvent. Following the $\frac{\epsilon+1}{\epsilon-1}$ dependence of the electrostatic potential in $\mathrm{PCM},{ }^{51,58}$ most of this shifting effect already occurs at small $\epsilon$ between 1 and 10, whereas a further increase of $\epsilon$ virtually does not affect the energies. As most of the solvents considered here have an $\epsilon$ above 20, this figure shows that the
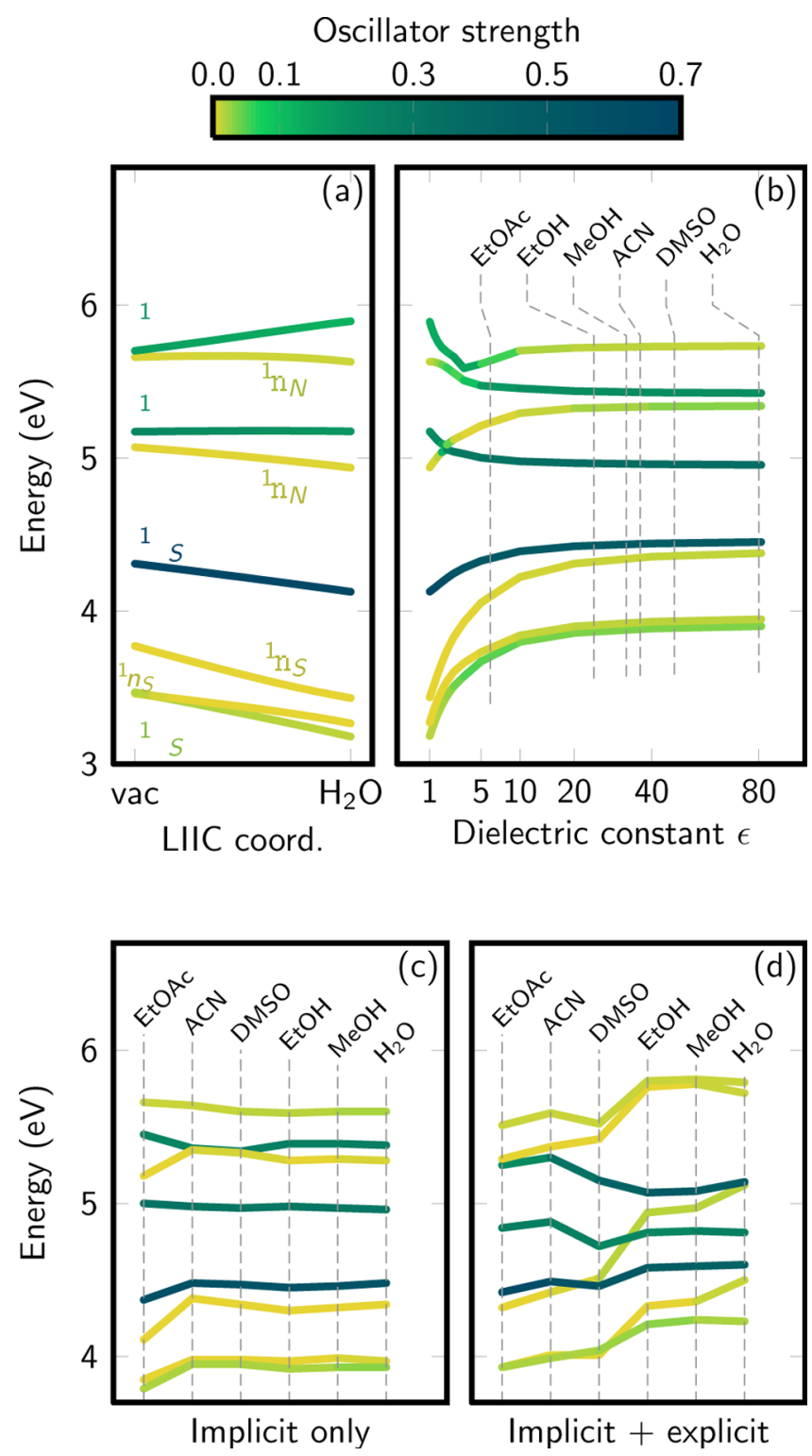

Figure 6. Decomposition of the solvatochromic shifts. In (a), the excitation energies and oscillator strengths (see color bar at the top) are shown for a linear interpolation in internal coordinates (LIIC) scan from the vacuum geometry to the geometry in $\mathrm{H}_{2} \mathrm{O}$ (taken from Figure 5f). In (b), at the latter geometry the dielectric constant $\epsilon$ is varied from 1 (vacuum) to $80\left(\mathrm{H}_{2} \mathrm{O}\right)$, with the refractive index kept at $n=1$. In (c), for each individual solvent the calculations considered the actual optimized geometries, $\epsilon$, and $n$, but no explicit solvent molecules (data in Table S7). In (d), additionally explicit solvation (as described above) is considered (data in Table 4).

solvatochromic effects on the excited states do not stem from the interaction between solvent dielectricity and state polarity.

In Figure 6c, we consider three solvent effects: geometry change, the dielectric constant, and additionally the refractive index. For each of the six solvents, in this panel we employ the actual optimized geometry in each solvent (not the one in water, as in panels (a) and (b)), the actual value of $\epsilon$ and the actual refractive index $n$. However, explicit solvent molecules are not included in panel (c). Interestingly, it can be seen that the excitation energies and oscillator strengths are fairly similar for all six solvents, with the exception of the less polar EtOAc solvent. As the experimental excitation energies and intensities 
vary considerably more than the results in panel (c), it can be concluded that the geometric relaxation and excited-state polarities are not the main causes for the differences observed between the solvents.

Finally, in panel (d) of Figure 6 we consider for each solvent all implicit and explicit solvent effects (geometry, $\epsilon, n$, and hydrogen bonds). It can be seen that the inclusion of the explicit solvent effects through microsolvation leads to significant differences in the excitation energies in the different solvents. In particular, the unpolar states are shifted to higher energies by large amounts in the polar protic solvents, whereas the bright states are less affected and move closer together. These findings agree with the results of the solvatochromic analysis, which also showed that the explicit solvent effects (hydrogen bonding) dominate the solvent absorption shifts in 2tCyt.

Solvatochromic Effects in 2tCyt. The experimental and computational results can now be put together to directly assign the features in the absorption spectra to the different electronic states. The most probable candidate for the electronic state responsible for the low-energy absorption maximum is the bright $S_{4}\left(\pi_{\mathrm{S}} \pi^{*}\right)$ state, based on the fact that its energy, oscillator strength, and solvent shifts all match with this absorption maximum. The only disagreement was found in the solvent-dependence of the band's intensity, with the experiment showing similar absorptivities in all solvents, whereas the calculations predict a lowering of the oscillator strength of $S_{4}$ in polar solvents. Interestingly, the $S_{4}$ is an example of a bright $\pi \pi^{*}$ state that blue-shifts in polar solvents, which is an unusual behavior for a $\pi \pi^{*}$ state of a nucleobase analogue. A reason for this behavior is the fact that the $S_{4}$ dipole moment is smaller than the ground state dipole moment of $2 \mathrm{tCyt}$. Additionally, based on the results in Figure 6 explicit solvent interactions are likely also involved in this blue-shift.

Likewise, we assign the second absorption maximum around 5.1-5.4 eV to the two $\pi \pi^{*}$ states localized on the pyrimidine ring $\left(S_{6}\right.$ and $\left.S_{8}\right)$, again based on a good match of the excitation energies, intensities, and solvent shifts. These two states show the typical behavior of a red-shift upon solvation in polar solvents, due to their very large dipole moments. Here, the calculations agree with experiment in that the band's intensity increases with increasing solvent polarity. Although the assignment of the weak high-energy shoulder cannot be done unambiguously, it may be associated with the upper $\pi \pi^{*}$ state $\left(S_{8}\right)$, since both move to lower energies in polar solvents.

The low-energy residual absorption tail can be at least partially attributed to the lowest $\pi_{S} \pi^{*}\left(S_{2}\right)$ and $n_{S} \pi^{*}\left(S_{1}\right)$ states, which in protic solvents is strongly blue-shifted and therefore becomes buried under the strong absorption of $S_{4}$, in line with the experimental observations. However, given the uncertainties in fitting the low-energy residual absorption next to the much brighter $S_{4}$ absorption and the phenomenological nature of the employed Gaussian deconvolution, we refrain from a more thorough analysis of the absorption tail. An alternative explanation for the absorption tail would be that it is due to the vibrational progression of the $S_{4}$ absorption, and that the different solvents lead to a different extent of this progression.

Generally, the excitation energy shifts from the vacuum calculation to the calculations including solvents can be explained partially by the dipole moment of the excited states, with less polar states being shifted to higher energies and more polar states to lower energies (compare the dipole moments in Table 3 ). The change of the ground state geometry in solution partially counteracts these shifts, but does not strongly affect the observations. More importantly, the specific solvent-solute interactions through hydrogen bonds appear to be responsible for the different shifts among the investigated solvents.

Comparison with Cytosine. 2tCyt exhibits solvatochromic trends roughly similar to cytosine (Cyt), which was theoretically investigated recently. ${ }^{52}$ For both nucleobases, the two lowest $n \pi^{*}$ states are destabilized by about $1 \mathrm{eV}$ in going from vacuum to water. The third $n \pi^{*}$ state is also predicted to significantly blue-shift in Cyt, but the destabilization is less drastic for $2 \mathrm{tCyt}$. For the fourth $n \pi^{*}$ state, the blue-shift is small $(0.1-0.3 \mathrm{eV})$ in both nucleobases. These similarities in the solvatochromic trends also extend to the $\pi \pi^{*}$ states. For Cyt, both the first and second $\pi \pi^{*}$ states are slightly $(0.1 \mathrm{eV})$ destabilized in going from vacuum to water. For $2 \mathrm{tCyt}$, the lowest $\pi \pi^{*}$ is more blue-shifted $(0.8 \mathrm{eV})$ while the second $\pi \pi^{*}$ state is only slightly destabilized $(0.3 \mathrm{eV})$. The third and fourth $\pi \pi^{*}$ states of Cyt and 2tCyt are red-shifted in going from vacuum to water, where the shifts are larger for $2 \mathrm{tCyt}$ than for Cyt.

Differences in the solvatochromic properties arise in the oscillator strengths of the $\pi \pi^{*}$ states. In $2 \mathrm{tCyt}$, the lowest $\pi \pi^{*}$ state is relatively dark $(0.01-0.03)$ with no observable solvent trend, while that of Cyt is bright and brightens in going from vacuum $(0.14)$ to water $(0.28)$. The second $\pi \pi^{*}$ state is bright for both molecules and darkens in going from vacuum to water. The third $\pi \pi^{*}$ state of Cyt brightens while that of $2 \mathrm{tCyt}$ darkens. The fourth $\pi \pi^{*}$ state brightens for both $2 \mathrm{tCyt}$ and Cyt. These different behaviors are probably due to different wave function characters of the Cyt vs $2 \mathrm{tCyt}$ states, and especially the participation of the two lowest $\pi^{*}$ orbitals.

Based on these results, it appears that 2tCyt and Cyt exhibit similar solvatochromic trends, where the lower-lying excited states tend to blue-shift (e.g., the lowest $\pi \pi^{*}$ and the two lowest $n \pi^{*}$ states) and only the higher $\pi \pi^{*}$ states red-shift in polar protic solvents. Another molecule related to $2 \mathrm{tCyt}$ and recently investigated theoretically is 5-methylcytidine, for which the first $\pi \pi^{*}$ and $n \pi^{*}$ states were shown ${ }^{59}$ to blue-shift when going from $\mathrm{THF} / \mathrm{ACN}$ to water, which is in line with the behavior of $2 \mathrm{tCyt}$ and Cyt.

\section{CONCLUSIONS}

In this contribution, we investigated the solvatochromic effects on the absorption spectrum of 2-thiocytosine (2tCyt) in six different solvents: ethyl acetate, dimethyl sulfoxide, acetonitrile, ethanol, methanol, and water. The absorption spectra generally show an intense absorption band at 269-286 nm, with an absorption tail extending to $330-360 \mathrm{~nm}$, depending on solvent. In acetonitrile, ethanol, methanol, and water, a second absorption maximum of comparable intensity was observed at $233-242 \mathrm{~nm}$, with a weak shoulder discernible at $210-218 \mathrm{~nm}$.

In order to identify the excited states responsible for the absorption bands and to explain the experimentally observed solvatochromic shifts, we used three popular solvatochromic scales and performed multistate complete-active-space perturbation theory computations for the only relevant tautomer of $2 \mathrm{tCyt}$, the 1-H-amino-thion form. These calculations included both explicit and implicit solvent effects on the electronic structure of $2 \mathrm{tCyt}$, by considering up to five solvent molecules of the first solvation shell and by means of a polarizable continuum model, respectively. The computations showed that, generally, the polarity of the solvent induces shifts in the excitation energies, with the unpolar states $S_{1}$ to $S_{5}$ being shifted 
to higher energies, and the more polar states $S_{6}$ to $S_{8}$ shifted to slightly lower energies. More importantly, the specific solutesolvent interactions through hydrogen bonds are mostly responsible for the differences in the absorption spectra in the different solvents. This finding is consistent with Catalán and Kamlet-Taft solvatochromic analyses of the lowest-energy absorption band, which showed that the solvatochromic parameter most relevant for this band is the hydrogen bond donor acidity of the solvent. Collectively, these findings imply that the absorption spectrum of $2 \mathrm{tCyt}$ responds sensitively to its microscopic environment, a finding that should aid in prospective applications of $2 \mathrm{tCyt}$ as a DNA/RNA photolabeling agent.

\section{ASSOCIATED CONTENT}

\section{S Supporting Information}

The Supporting Information is available free of charge on the ACS Publications website at DOI: 10.1021/acs.jpcb.7b02715.

Further details and discussions on the level of theory, details of the Gaussian fit, full solvatochromic analyses, and molecular coordinates from Figure 5 (PDF)

\section{AUTHOR INFORMATION}

\section{Corresponding Authors}

*E-mail: carlos.crespo@case.edu.

*E-mail: leticia.gonzalez@univie.ac.at.

ORCID

Carlos E. Crespo-Hernández: 0000-0002-3594-0890

Leticia González: 0000-0001-5112-794X

Notes

The authors declare no competing financial interest.

\section{ACKNOWLEDGMENTS}

S.M., P.M., and L.G. thank the Austrian Science Fund (FWF) through project $\mathrm{P} 25827$, the COST action CM1405 (MOLIM), and the Vienna Scientific Cluster (VSC) for the allocation of computational time. B.A. and C.E.C.-H. acknowledge the CAREER program of the National Science Foundation (Grants No. CHE-1255084 and CHE-1539808) for financial support. The authors also thank Lara MartínezFernández and Inés Corral for preliminary discussions, and Marvin Pollum for comments and discussions on early versions of the manuscript.

\section{REFERENCES}

(1) Carell, T.; Brandmayr, C.; Hienzsch, A.; Müller, M.; Pearson, D.; Reiter, V.; Thoma, I.; Thumbs, P.; Wagner, M. Structure and Function of Noncanonical Nucleobases. Angew. Chem., Int. Ed. 2012, 51, 71107131.

(2) Périgaud, C.; Gosselin, G.; Imbach, J. L. Nucleoside Analogues as Chemotherapeutic Agents: A Review. Nucleosides Nucleotides 1992, 11, 903-945.

(3) Sampath, D.; Rao, V. A.; Plunkett, W. Mechanisms of Apoptosis Induction by Nucleoside Analogs. Oncogene 2003, 22, 9063-9074.

(4) Jordheim, L. P.; Durantel, D.; Zoulim, F.; Dumontet, C. Advances in the Development of Nucleoside and Nucleotide Analogues for Cancer and Viral Diseases. Nat. Rev. Drug Discovery 2013, 12, 447-464.

(5) Mitsuya, H.; Yarchoan, R.; Broder, S. Molecular Targets for AIDS Therapy. Science 1990, 249, 1533-1544.

(6) Ajitkumar, P.; Cherayil, J. D. Thionucleosides in Transfer Ribonucleic Acid: Diversity, Structure, Biosynthesis, and Function. Microbiol. Rev. 1988, 52, 103-113.
(7) Carbon, J.; David, H.; Studier, M. H. Thiobases in Escherchia coli Transfer RNA: 2-Thiocytosine and 5-Methylaminomethyl-2-thiouracil. Science 1968, 161, 1146-1147.

(8) Yamada, Y.; Saneyoshi, M.; Nishimura, S. Isolation and Characterization of 2-Thiocytidine from a Serine Transfer Ribonucleic Acid of Escherichia coli. FEBS Lett. 1970, 7, 207-210.

(9) Wilhelmsson, L. M. Fluorescent Nucleic Acid Base Analogues. Q. Rev. Biophys. 2010, 43, 159-183.

(10) Sinkeldam, R. W.; Greco, N. J.; Tor, Y. Fluorescent Analogs of Biomolecular Building Blocks: Design, Properties, and Applications. Chem. Rev. 2010, 110, 2579-2619.

(11) Favre, A.; Saintomé, C.; Fourrey, J.-L.; Clivio, P.; Laugâa, P. Thionucleobases as Intrinsic Photoaffinity Probes of Nucleic Acid Structure and Nucleic Acid-Protein Interactions. J. Photochem. Photobiol., B 1998, 42, 109-124.

(12) Massey, A.; Xu, Y.-Z.; Karran, P. Photoactivation of DNA Thiobases as a Potential Novel Therapeutic Option. Curr. Biol. 2001, 11, 1142-1146.

(13) Karran, P.; Attard, N. Thiopurines in Current Medical Practice: Molecular Mechanisms and Contributions to Therapy-Related Cancer. Nat. Rev. Cancer 2008, 8, 24-36.

(14) Pridgeon, S. W.; Heer, R.; Taylor, G. A.; Newell, D. R.; O’Toole, K.; Robinson, M.; Xu, Y.-Z.; Karran, P.; Boddy, A. V. Thiothymidine Combined with UVA as a Potential Novel Therapy for Bladder Cancer. Br. J. Cancer 2011, 104, 1869-1876.

(15) Pollum, M.; Jockusch, S.; Crespo-Hernández, C. E. Increase in the Photoreactivity of Uracil Derivatives by Doubling Thionation. Phys. Chem. Chem. Phys. 2015, 17, 27851-27861.

(16) Pollum, M.; Martínez-Fernández, L.; Crespo-Hernández, C. E. In Photoinduced Phenomena in Nucleic Acids I, Top. Curr. Chem.; Barbatti, M., Borin, A. C., Ullrich, S., Eds.; Springer Berlin Heidelberg, 2015; Vol. 355, pp 245-327.

(17) Mai, S.; Pollum, M.; Martínez-Fernández, L.; Dunn, N.; Marquetand, P.; Corral, I.; Crespo-Hernández, C. E.; González, L. The Origin of Efficient Triplet State Population in Sulfur-Substituted Nucleobases. Nat. Commun. 2016, 7, 13077.

(18) Crespo-Hernández, C. E.; Cohen, B.; Hare, P. M.; Kohler, B. Ultrafast Excited-State Dynamics in Nucleic Acids. Chem. Rev. 2004, 104, 1977-2020.

(19) Mai, S.; Richter, M.; Marquetand, P., González, L. In Photoinduced Phenomena in Nucleic Acids I, Top. Curr. Chem.; Barbatti, M., Borin, A. C., Ullrich, S., Eds.; Springer Berlin Heidelberg, 2015; Vol. 355, pp 99-153.

(20) Cuervo, A.; Dans, P. D.; Carrascosa, J. L.; Orozco, M.; Gomila, G.; Fumagalli, L. Direct Measurement of the Dielectric Polarization Properties of DNA. Proc. Natl. Acad. Sci. U. S. A. 2014, 111, E3624E3630.

(21) Mautner, G.; Kindt-Larsen, T.; Bergson, G.; Andersen, I. G. K.; Munch-Petersen, J. Some Remarks on Position-Conditioned Differences of the Ultraviolet Spectra and Chemical Reactivities of Disubstituted Pyrimidines and Purines. Acta Chem. Scand. 1963, 17, 1694-1704.

(22) Sinsheimer, R. L.; Scott, J. F.; Loofbourow, J. R. Ultraviolet Absorption Spectra at Reduced Temperature II. Pyrimidines and Purines. J. Biol. Chem. 1950, 187, 313-324.

(23) Brown, D. J.; Teitei, T. Simple Pyrimidines VIII. The Fine Structure of Isocytosine, Thiocytosine and Some Isomers. Aust. J. Chem. 1965, 18, 559-568.

(24) Igarashi-Yamamoto, N.; Tajiri, A.; Hatano, M.; Shibuya, S.; Ueda, T. Ultraviolet Absorption, Circular Dichroism and Magnetic Circular Dichroism Studies of Sulfur-Containing Nucleic Acid Bases and Their Nucleosides. Biochim. Biophys. Acta, Nucleic Acids Protein Synth. 1981, 656, 1-15.

(25) Civcir, P. U. AM1 and PM3 Studies of Some Thio Analogues of Pyrimidine Bases in the Gas and Aqueous Phases. J. Phys. Org. Chem. 2001, 14, 171-179.

(26) Contreras, J. G.; Alderete, J. B. Aqueous Solvation Effect on the Prototropic Tautomerism of 2-Thiocytosine. J. Phys. Org. Chem. 1995, 8, 395-399. 
(27) Contreras, J.; Alderete, J. B.; Gnecco, J. A. Semiempirical Molecular Orbital Calculations on the Prototropic Tautomerism of 2 Thiocytosine. J. Mol. Struct.: THEOCHEM 1991, 251, 195-204.

(28) Leś, A.; Ortega-Blake, I. Tautomerism of Uracil, Cytosine, Isocytosine, and Some of their Thio-Derivatives. Int. J. Quantum Chem. 1986, 30, 225-237.

(29) Kwiatkowski, J. S.; Leszczyński, J. Molecular Structure and Vibrational IR Spectra of Cytosine and Its Thio and Seleno Analogues by Density Functional Theory and Conventional ab Initio Calculations. J. Phys. Chem. 1996, 100, 941-953.

(30) Latosińska, J. N.; Seliger, J.; Žagar, V.; Burchardt, D. V. A Comparative Study of the Hydrogen-Bonding Patterns and Prototropism in Solid 2-Thiocytosine (Potential Antileukemic Agent) and Cytosine, as Studied by $1 \mathrm{H}-14 \mathrm{~N}$ NQDR and QTAIM/ DFT. J. Mol. Model. 2012, 18, 11-26.

(31) Podolyan, Y.; Gorb, L.; Blue, A.; Leszczynski, J. A Theoretical Investigation of Tautomeric Equilibria and Proton Transfer in Isolated and Hydrated Thiocytosine. J. Mol. Struct.: THEOCHEM 2001, 549, 101-109.

(32) Rostkowska, H.; Nowak, M. J.; Lapinski, L.; Bretner, M.; Kulikowski, T.; Les, A.; Adamowicz, L. Theoretical and MatrixIsolation Experimental Studies on 2-Thiocytosine and 5-Fluoro-2Thiocytosine. Biochim. Biophys. Acta, Gene Struct. Expression 1993, 1172, 239-246.

(33) Catalán, J. Toward a Generalized Treatment of the Solvent Effect Based on Four Empirical Scales: Dipolarity (SdP, a New Scale), Polarizability (SP), Acidity (SA), and Basicity (SB) of the Medium. J. Phys. Chem. B 2009, 113, 5951-5960.

(34) Kamlet, M. J.; Abboud, J. L. M.; Abraham, M. H.; Taft, R. W. Linear Solvation Energy Relationships. 23. A Comprehensive Collection of the Solvatochromic Parameters, $\pi^{*}, \alpha$, and $\beta$, and Some Methods for Simplifying the Generalized Solvatochromic Equation. J. Org. Chem. 1983, 48, 2877-2887.

(35) Reichardt, C. Solvatochromic Dyes as Solvent Polarity Indicators. Chem. Rev. 1994, 94, 2319-2358.

(36) Kossmann, S.; Neese, F. Efficient Structure Optimization with Second-Order Many-Body Perturbation Theory: The RIJCOSX-MP2 Method. J. Chem. Theory Comput. 2010, 6, 2325-2338.

(37) Dunning, T. H. Gaussian Basis Sets for Use in Correlated Molecular Calculations. I. The Atoms Boron Through Neon and Hydrogen. J. Chem. Phys. 1989, 90, 1007-1023.

(38) Woon, D. E.; Dunning, T. H. Gaussian Basis Sets for Use in Correlated Molecular Calculations. III. The Atoms Aluminum Through Argon. J. Chem. Phys. 1993, 98, 1358-1371.

(39) Sinnecker, S.; Rajendran, A.; Klamt, A.; Diedenhofen, M.; Neese, F. Calculation of Solvent Shifts on Electronic g-Tensors with the Conductor-Like Screening Model (COSMO) and Its SelfConsistent Generalization to Real Solvents (Direct COSMO-RS). J. Phys. Chem. A 2006, 110, 2235-2245.

(40) Neese, F. The ORCA Program System. WIREs Comput. Mol. Sci. 2012, 2, 73-78.

(41) Finley, J.; Malmqvist, P. Å.; Roos, B. O.; Serrano-Andrés, L. The Multi-State CASPT2 Method. Chem. Phys. Lett. 1998, 288, 299-306.

(42) Widmark, P.-O.; Malmqvist, P.-Å.; Roos, B. O. Density-Matrix Averaged Atomic Natural Orbital (ANO) Basis-Sets for Correlated Molecular Wave-Functions. Theor. Chim. Acta 1990, 77, 291-306.

(43) Roos, B. O.; Lindh, R.; Malmqvist, P. Å.; Veryazov, V.; Widmark, P.-O. Main Group Atoms and Dimers Studied with a New Relativistic ANO Basis Set. J. Phys. Chem. A 2004, 108, 2851-2858.

(44) Reiher, M. Relativistic Douglas-Kroll-Hess Theory. WIREs Comput. Mol. Sci. 2012, 2, 139-149.

(45) Aquilante, F.; Lindh, R; Bondo Pedersen, T. Unbiased Auxiliary Basis Sets For Accurate Two-Electron Integral Approximations. J. Chem. Phys. 2007, 127, 114107.

(46) Ghigo, G.; Roos, B. O.; Malmqvist, P. Å. A Modified Definition of the Zeroth-Order Hamiltonian in Multiconfigurational Perturbation Theory (CASPT2). Chem. Phys. Lett. 2004, 396, 142-149.

(47) Zobel, J. P.; Nogueira, J.; González, L. The IPEA Dilemma in CASPT2. Chem. Sci. 2017, 8, 1482-1499.
(48) Aquilante, F.; Autschbach, J.; Carlson, R. K.; Chibotaru, L. F.; Delcey, M. G.; De Vico, L.; Fdez Galván, I.; Ferré, N.; Frutos, L. M.; Gagliardi, L.; et al. Molcas 8: New Capabilities for Multiconfigurational Quantum Chemical Calculations Across the Periodic Table. J. Comput. Chem. 2016, 37, 506-541.

(49) Neese, F. An Improvement of the Resolution of the Identity Approximation for the Formation of the Coulomb Matrix. J. Comput. Chem. 2003, 24, 1740-1747.

(50) TURBOMOLE V7.0, A development of University of Karlsruhe and Forschungszentrum Karlsruhe $\mathrm{GmbH}, 2015$.

(51) Serrano-Andrés, L.; Fülscher, M. P.; Karlström, G. Solvent Effects on Electronic Spectra Studied by Multiconfigurational Perturbation Theory. Int. J. Quantum Chem. 1997, 65, 167-181.

(52) Li, Q.; Mennucci, B.; Robb, M. A.; Blancafort, L.; Curutchet, C. Polarizable QM/MM Multiconfiguration Self-Consistent Field Approach with State-Specific Corrections: Environment Effects on Cytosine Absorption Spectrum. J. Chem. Theory Comput. 2015, 11, $1674-1682$.

(53) Mennucci, B. Modeling Absorption and Fluorescence Solvatochromism with QM/Classical Approaches. Int. J. Quantum Chem. 2015, 115, 1202-1208.

(54) Forsberg, N.; Malmqvist, P. Å. Multiconfiguration Perturbation Theory with Imaginary Level Shift. Chem. Phys. Lett. 1997, 274, 196204.

(55) Avila Ferrer, F. J.; Cerezo, J.; Stendardo, E.; Improta, R.; Santoro, F. Insights for an Accurate Comparison of Computational Data to Experimental Absorption and Emission Spectra: Beyond the Vertical Transition Approximation. J. Chem. Theory Comput. 2013, 9, 2072-2082.

(56) Taft, R. W.; Kamlet, M. J. The Solvatochromic Comparison Method. 2. The $\alpha$-Scale of Solvent Hydrogen-Bond Donor (HBD) Acidities. J. Am. Chem. Soc. 1976, 98, 2886-2894.

(57) Catalán, J. On the ET(30), $\pi^{*}$, Py, S', and SPP Empirical Scales as Descriptors of Nonspecific Solvent Effects. J. Org. Chem. 1997, 62, 8231-8234.

(58) Tomasi, J.; Mennucci, B.; Cammi, R. Quantum Mechanical Continuum Solvation Models. Chem. Rev. 2005, 105, 2999-3094.

(59) Martínez-Fernández, L.; Pepino, A. J.; Segarra-Martí, J.; Banyasz, A.; Garavelli, M.; Improta, R. Computing the Absorption and Emission Spectra of 5-Methylcytidine in Different Solvents: A Test-Case for Different Solvation Models. J. Chem. Theory Comput. 2016, 12, 44304439. 